\title{
The protective effect of puerarin on angiotensin II-induced aortic aneurysm formation by the inhibition of NADPH oxidase activation and oxidative stress-triggered AP-1 signaling pathways
}

\author{
JIE YUE ${ }^{1,2}$, SHUNWU CHANG ${ }^{3}$, ZHANXIANG XIAO $^{4}$, YOUFEI QI ${ }^{4}$ and JIANXING HE ${ }^{1,2}$ \\ ${ }^{1}$ The First Clinical College, Southern Medical University, Guangzhou, Guangdong 510515; ${ }^{2}$ Department of Cardiothoracic \\ Surgery, The First Affiliated Hospital of Guangzhou Medical University; Departments of ${ }^{3}$ Gastrointestinal Surgery \\ and ${ }^{4}$ Vascular Surgery, Hainan Provincial People's Hospital, Guangzhou, Guangdong 510120, P.R. China
}

Received April 6, 2016; Accepted April 5, 2018

DOI: $10.3892 / \mathrm{ol} .2018 .9021$

\begin{abstract}
Puerarin, an active ingredient of Pueraria lobata, has a range of pharmacological effects and excellent pharmacodynamic properties. In the present study, the effect of puerarin on angiotensin II-induced aortic aneurysm formation and the potential underlying molecular mechanisms were examined. The results revealed that puerarin significantly suppressed the viability, and induced the apoptosis, of aneurysm-inducing cells in a time- and dose-dependent manner. Furthermore, treatment with puerarin significantly suppressed the production of reactive oxygen species (ROS) and the expression of matrix metalloproteinase-2 (MMP-2) protein in aneurysm cells. Puerarin treatment significantly increased caspase- 9 and -3 activity, induced the protein expression of phosphorylated (p)-Jun and inhibited the protein expression of activator protein 1 (AP-1) in aneurysm cells. It was also demonstrated that Puerarin significantly suppressed the reduced nicotinamide-adenine dinucleotide phosphate (NADPH) oxidase activity in aneurysm cells. Therefore, it was demonstrated that puerarin on suppressed the cell growth of angiotensin II-induced aortic aneurysm formation by affecting the rate of apoptosis, the generation of ROS, MMP-2, AP-1 and p-Jun protein expression and NADPH oxidase.
\end{abstract}

\section{Introduction}

Abdominal aortic aneurysm (AAA) refers to the local permanent expansion of the aorta, and is defined as an aortic diameter $>1.5$ times larger than the normal value (typically $>3 \mathrm{~cm}$ ).

Correspondence to: Mr. Jianxing He, Department of Cardiothoracic Surgery, The First Affiliated Hospital of Guangzhou Medical University, 151 Yanjiang Road, Guangzhou, Guangdong 510120, P.R. China

E-mail: jiashaba0763722334@126.com

Key words: puerarin, aortic aneurysm, reduced nicotinamide-adenine dinucleotide phosphate oxidase activity, activator protein 1
Aortectasia refers to the local expansion of the aorta with a diameter $<1.5$ times of the normal value (1). The morbidity of AAA for individuals $>65$ years is up to $9 \%$ (2). The majority of arterial aneurysms are asymptomatic; however, damage to the vascular structure of the aorta subsequent to aneurysm will lead to an increase in the aorta tube diameter (3). If the diameter is $>5.5 \mathrm{~cm}$, it is likely to eventually rupture; the risk of aortoclasia is associated with the diameter size (4). The prognosis for AAA ruptures remains poor, with a mortality rate of $80-90 \%$ (4). The primary therapeutic strategy for AAA with a diameter $>5.5 \mathrm{~cm}$ includes surgery and intracavitary interventional treatment (5). However, for AAA with a diameter $<5.5 \mathrm{~cm}$, no effective therapy option is available (5).

Inflammatory cell infiltration is a critical event in the formation of AAA. Inflammatory cells may secrete a large number of inflammatory cytokines, chemokines, chemotax is cytokines, leukotrienes, reactive oxygen species (ROS) and immunoglobulins (6). The vasa vasorum of the aorta is the channel for inflammatory cell intrusion into the aortic intima and membrane (6). Mesolamella neovascularization formation and vascular smooth muscle cell reduction are characteristics of AAA (7). An intraluminal thrombus may induce neovascularization and inflammation by inducing hypoxia in the endometrium and membrane. Inflammatory cells in the thrombus may release proteases, including matrix metalloproteinase 9 (MMP-9) and urokinase-type fibrinolytic enzymes (8). Inflammation of AAA is primarily induced by cytokines expressed by type $2 \mathrm{~T}$ helper cells (8); however, there may also be cytokines from type $1 \mathrm{~T}$ helper cells (8). In addition, ROS and reactive nitrogen species function in a number of types of chronic disease. An increase in ROS is associated with a local inflammatory reaction that may lead to cell and tissue damage, i.e. oxidative stress (9). A previous study demonstrated the effects of oxidative stress on the pathogenesis of AAA (9).

Puerarin (Fig. 1) is an active ingredient abundant in Pueraria lobata (10). Puerarin demonstrates a variety of pharmacological activities and specific pesticide effects; in particular, puerarin has demonstrated efficacy in the treatment of cardiovascular and cerebrovascular diseases (11). Puerarin is a promising medicine with the potential for a variety of 
clinical applications, as is effective despite low toxicity and few side effects (12). A previous study demonstrated that puerarin exerts an endothelium-dependent dilation effect (12). Puerarin mediated ion channels in smooth muscle cells have been demonstrated to improve its water solubility and lipid solubility, enhance combination capacity between drugs and hemoglobin, reduce drug polarity, improve oral bioavailability and drug targeting effects $(13,14)$.

In the present study, it was examined whether puerarin exhibited an anti-inflammation effect. The results of the present study demonstrated that puerarin significantly inhibited cell growth in angiotensin II (angII)-induced aortic aneurysm formation by activating reduced nicotinamide-adenine dinucleotide phosphate (NADPH) oxidase and oxidative stress-induced activator protein 1 (AP-1) signaling pathways.

\section{Materials and methods}

Ethical statement and animals. C57BL/6 background mice ( $n=24$, male, 5-6 weeks, 20-22 g) were obtained from the Experiment Center of The First Affiliated Hospital of Guangzhou Medical University (Guangzhou, China) and housed at $22-24^{\circ} \mathrm{C}, 55-60 \%$ humidity, $12 \mathrm{~h}$ light/dark cycle, and free access to food and water. All mice were randomly divided into the following 4 experimental groups: Sham $(\mathrm{n}=6)$, angII model $(\mathrm{n}=6), 100 \mathrm{mg} / \mathrm{kg}$ puerarin treatment $(\mathrm{n}=6)$ and $200 \mathrm{mg} / \mathrm{kg}$ puerarin treatment $(\mathrm{n}=6)$ groups. Mice from the angII model, $100 \mathrm{mg} / \mathrm{kg}$ puerarin treatment and $200 \mathrm{mg} / \mathrm{kg}$ puerarin treatment groups were continuously treated with angII $(1,000 \mathrm{ng} / \mathrm{kg} / \mathrm{min})$ for 4 weeks, as previously described (15). From the third week onwards, mice from the 100 and $200 \mathrm{mg} / \mathrm{kg}$ puerarin treatment groups were continuously administered with 100 or $200 \mathrm{mg} / \mathrm{kg}$ puerarin for 2 weeks, respectively. All protocols in the present study were approved by the Ethics Committee of the Hainan Provincial People's Hospital (Guangzhou, China). Following treatment with puerarin, mice were anesthetizated using $35 \mathrm{mg} / \mathrm{kg}$ pentobarbital sodium, and then sacrificed using decollation. The tissue of aortic aneurysm was acquired and stored at $-80^{\circ} \mathrm{C}$.

Determination of the activity of NADPH oxidase. Proteins were extracted from isolated abdominal aortic tissues homogenized using PTN50 buffer (50 mM Na $\mathrm{PO}_{4}, \mathrm{pH} 7.4,50 \mathrm{mM} \mathrm{NaCl}$ and $1 \%$ Triton $\mathrm{X}-100$ ) with protease inhibitors (cat. no. P8340; Sigma-Aldrich; Merck KGaA, Darmstadt, Germany) at $4^{\circ} \mathrm{C}$ for $15 \mathrm{~min}$. A bicinchoninic acid (BCA) kit (Pierce; Thermo Fisher Scientific, Inc., Waltham, MA, USA) was used to determine protein concentration. NADPH oxidase activity was assessed using a NADPH oxidase activity kit (catalog no. A127, Nanjing Jiancheng Biology Engineering Institute) in a 50-mmol/1 phosphate buffer ( $\mathrm{pH} 7.0$ ) containing $100 \mu \mathrm{mol} / 1$ NADPH as the substrate. Photoemissions were then detected using the Lumat LB9507 luminometer (Berthold Technologies GmbH \& Co. KG, Bad Wildbad, Germany).

Detection of ROS production. Isolated abdominal aortic tissues were homogenized with a homogenizer for $20 \mathrm{sec}$ in $2 \mathrm{ml}$ RIPA assay (Beyotime Institute of Biotechnology, Nanjing, China). An aliquot of 2'7'-dichlorodihydroflurescein diacetate $(25 \mu \mathrm{mol} / \mathrm{l})$ was added to the homogenates and ROS production was determined by absorbance at $530 \mathrm{~nm}$ using an ELISA plate reader (Bio-Rad Laboratories, Inc., Hercules, CA, USA).

Western blot analysis. Proteins were extracted from isolated abdominal aortic tissues homogenized using PTN50 buffer with protease inhibitors, as previously described. The BCA kit was used to determine protein concentration. A total of $60 \mu \mathrm{g}$ protein/lane was separated using 12\% SDS-PAGE and transferred to a polyvinylidene difluoride membrane (Invitrogen; Thermo Fisher Scientific, Inc.). The membrane was blocked using 5\% skimmed milk in PBS-Tween-20 (0.05\%) for $1 \mathrm{~h}$ at $37^{\circ} \mathrm{C}$ and immunoblotted using anti-MMP-2 (sc-10736; dilution, 1:3,000), anti-AP-1 (sc-52; dilution, 1:3,000), and anti- $\beta$-actin (dilution, 1:3,000; all Santa Cruz Biotechnology, Inc., Dallas, TX, USA) and anti-phosphorylated-Jun (p-Jun; 3270; dilution, 1:4,000; Cell Signaling Technology, Inc., Danvers, MA, USA) antibodies overnight at $4^{\circ} \mathrm{C}$. Subsequently, the membrane was incubated with horseradish peroxidase-conjugated rabbit anti-goat secondary antibody (sc-2004 dilution, 1:5,000; Santa Cruz Biotechnology, Inc.) at $37^{\circ} \mathrm{C}$ for $1 \mathrm{~h}$, detected using an enhanced chemiluminescence substrate (GE Healthcare, Chicago, IL, USA) and analyzed using Image Lab version 3.0 (Bio-Rad Laboratories, Inc., Hercules, CA, USA).

Cell culture and proliferation. Murine hemangioendothelioma (EOMA) cells were obtained from the Shanghai Cell Bank (Shanghai, China) and cultured with Dulbecco's modified Eagle's medium (Thermo Fisher Scientific, Inc.) containing $10 \%$ fetal bovine serum (Thermo Fisher Scientific, Inc.) at $37^{\circ} \mathrm{C}$ in an atmosphere containing $5 \% \mathrm{CO}_{2}$. EOMA cells were plated at 3,000 cells/well in 96-well plates and cultured with dimethyl sulfoxide (DMSO; Thermo Fisher Scientific, Inc.), 50,100 or $200 \mu \mathrm{M}$ puerarin for 0,24 or $48 \mathrm{~h}$. Subsequently, $150 \mu \mathrm{l}$ MTT (Thermo Fisher Scientific, Inc.) was added to each well and incubated at room temperature for $4 \mathrm{~h}$. The medium was removed and $200 \mu \mathrm{l}$ of DMSO (Thermo Fisher Scientific, Inc.) was added into each well for $20 \mathrm{~min}$ to dissolve formazan. The cell viability rate was determined at $490 \mathrm{~nm}$ using an ELISA plate reader.

Apoptosis rate determination. EOMA cells were plated at $1 \times 10^{6}$ cells/well in 6-well plates and cultured with DMSO, or 50,100 or $200 \mu \mathrm{M}$ puerarin for $48 \mathrm{~h}$. EOMA cells were stained with $10 \mu \mathrm{l}$ annexin $\mathrm{V}$-allophycocyanin (eBioscience; Thermo Fisher Scientific, Inc.) and $5 \mu$ propidium iodide at room temperature in the dark for $30 \mathrm{~min}$. The rate of apoptosis was determined using flow cytometry $\left(\right.$ EPICS $^{\circledR}$ ALTRA $^{\text {TM }}$; Beckman Coulter, Inc., Brea, CA, USA) and analyzed using FlowJo version 7.6.1 (FlowJo LLC, Ashland, OR, USA).

Detection of caspase-9/3 activity. EOMA cells were plated at $1 \times 10^{6}$ cells/well in 6-well plates and cultured with DMSO, or 50, 100 or $200 \mu \mathrm{M}$ puerarin for $48 \mathrm{~h}$. EOMA cells were stained with Ac-LEHD-pNA (Beyotime Institute of Biotechnology) for caspase-9 activity and Ac-DEVD- $p$ NA (Beyotime Institute of Biotechnology) for caspase- 3 activity at $37^{\circ} \mathrm{C}$ for $1 \mathrm{~h}$. The caspase-9/3 activity was determined at the wavelength of $405 \mathrm{~nm}$ using an ELISA plate reader. 


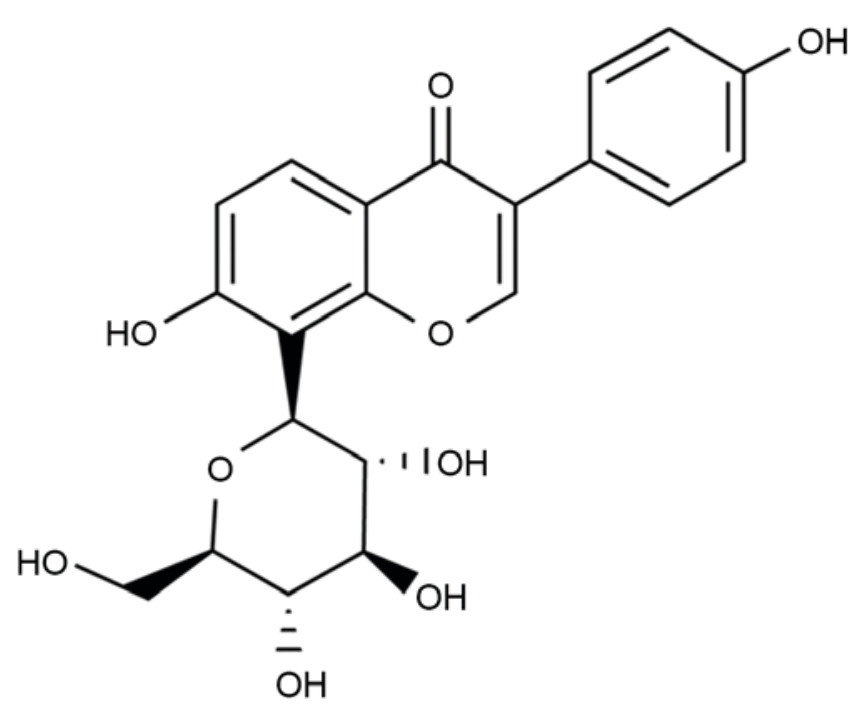

Figure 1. Molecular structure of puerarin.

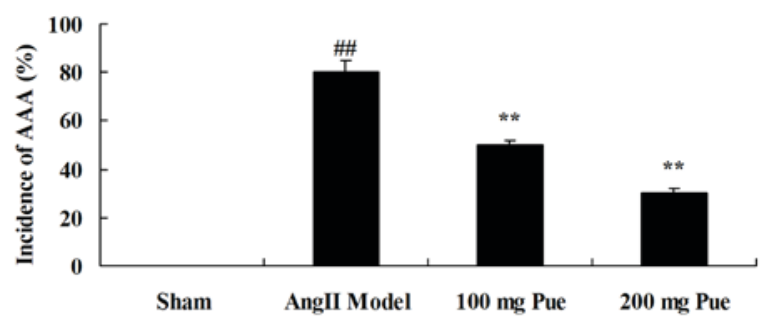

Figure 2. Pue treatment attenuated angII-induced AAA in mice. ${ }^{\# \#} \mathrm{P}<0.01$ vs. sham group; ${ }^{*} \mathrm{P}<0.05,{ }^{* *} \mathrm{P}<0.01$ vs. angII model group. Pue, puerarin; AAA, abdominal aortic aneurysm; angII, angiotensin II.

Statistical analysis. The data are expressed as the mean \pm standard error of the mean using SPSS version 17.0 (SPSS, Inc., Chicago, IL, USA). Statistical analysis was performed with an analysis of variance followed by Tukey's post hoc test. $\mathrm{P}<0.05$ was considered to indicate a statistically significant difference.

\section{Results}

Puerarin attenuates angII-induced AAA in mice. AngII-induced AAA was initially observed in the different groups. As presented in Fig. 2, the angII-induced AAA rate of the AAA model was markedly increased, compared with that of the sham control group. However, puerarin decreased the angII-induced AAA rate in mice (Fig. 2).

Puerarin suppresses the NADPH oxidase activity in angII-induced AAA in mice. Subsequently, the NADPH oxidase activity from each group was determined, to analyze the protective effect of puerarin on the formation of angII-induced aortic aneurysms. Compared with the sham group, NADPH oxidase activity was observably increased by angII (Fig. 3). Treatment with puerarin significantly decreased the angII-induced NADPH oxidase activity in mice (Fig. 3).

Puerarin suppresses MMP-2 protein expression in angII-induced $A A A$ in mice. It was hypothesized that an

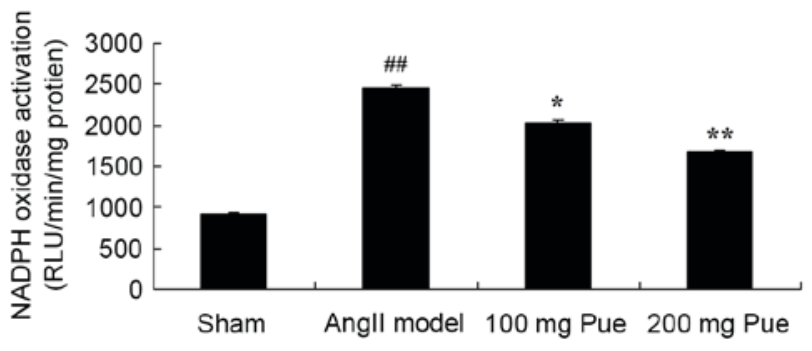

Figure 3. Pue treatment suppressed the NADPH oxidase activity in angII-induced AAA in mice. ${ }^{\# /} \mathrm{P}<0.01$ vs. sham group; ${ }^{*} \mathrm{P}<0.05,{ }^{* * *} \mathrm{P}<0.01$ vs. angII model group. Pue, puerarin; NADPH, reduced nicotinamide-adenine dinucleotide phosphate; angII, angiotensin II; AAA, abdominal aortic aneurysm; RLU, relative light units.

effect on MMP-2 protein expression was important in the protective effect of puerarin on angII-induced aortic aneurysm formation. The results from western blot analysis demonstrated that angII markedly induced MMP-2 protein expression in the AAA model group compared with the sham group (Fig. 4). Puerarin treatment significantly inhibited the expression of MMP-2 protein in angII-induced AAA in mice (Fig. 4).

Puerarin inhibits the AP-1 protein expression in angII-induced $A A A$ in mice. The effect of puerarin on the AP-1 protein expression of angII-induced AAA in mice was analyzed. As presented in Fig. 4, angII significantly induced AP-1 protein expression in AAA mice compared with the sham group. Treatment with puerarin significantly inhibited the AP-1 protein expression of angII-induced AAA in mice (Fig. 4).

Puerarin induces the $p$-Jun protein expression of angII-induced AAA in mice. Western blot analysis was used to determine the effect of puerarin on p-Jun protein expression in angII-induced AAA in mice. As presented in Fig. 4, p-Jun protein expression of AAA in mice was significantly suppressed by angII compared with the sham mice. Puerarin treatment significantly increased the p-Jun protein expression of AAA in mice (Fig. 4).

Puerarin suppresses the ROS production in angII-induced $A A A$ in mice. Subsequently, whether puerarin suppressed the ROS production of angII-induced AAA in mice was investigated. As presented in Fig. 5, there was a marked increase in ROS production of angII-induced AAA model mice compared with the sham group. Puerarin treatment significantly decreased the extent of ROS production in angII-induced AAA mice (Fig. 5).

Puerarin attenuates the viability of EOMA cells. A total of 50, 100 or $200 \mu \mathrm{M}$ puerarin was added to EOMA cells and viability was determined using an MTT assay. As presented in Fig. 6, puerarin significantly suppressed the viability of EOMA cells, compared with the sham group (DMSO only).

Puerarin induces the apoptosis of EOMA cells. The effect of puerarin on the apoptosis of EOMA cells was subsequently determined. As presented in Fig. 7, puerarin induced the apoptosis of EOMA cells in a dose-dependent manner. In 
A

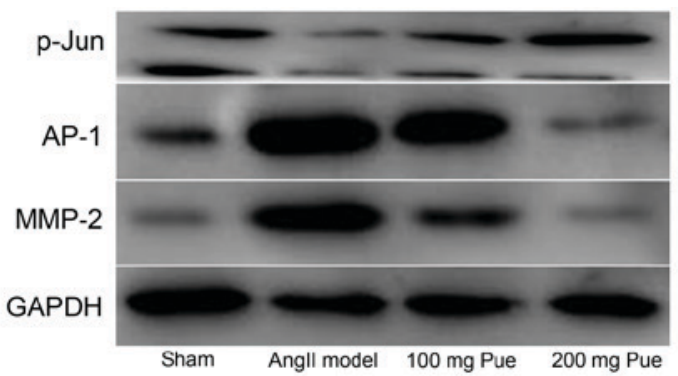

C

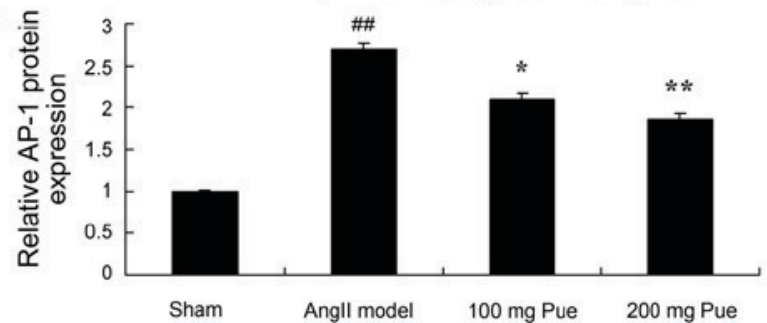

B

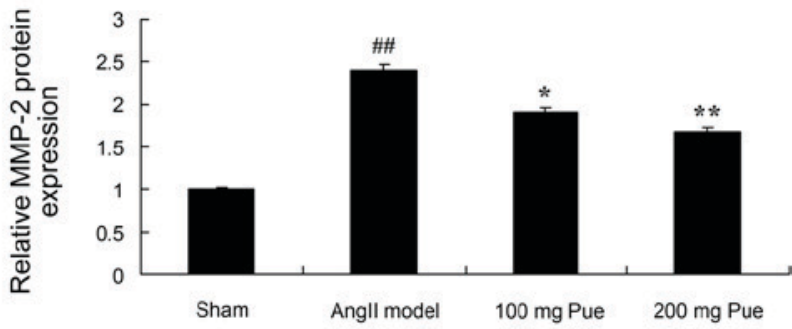

D

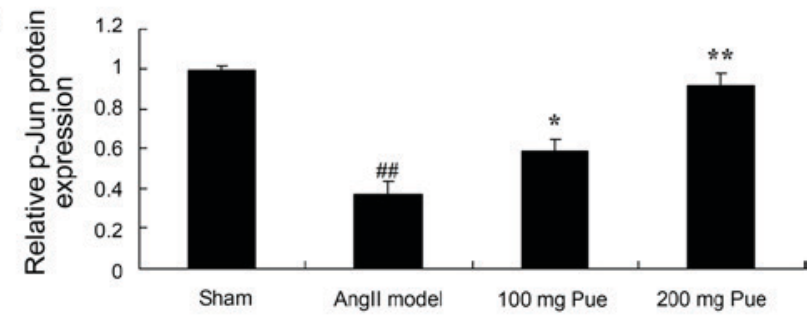

Figure 4. Pue treatment suppressed MMP-2 and AP-1 protein expression, and promoted p-Jun protein expression, in angII-induced abdominal aortic aneurysm in mice. (A) Western blotting was performed, and the results for (B) MMP-2, (C) AP-1 and (D) p-Jun were quantified by normalization to GAPDH. ${ }^{\# \#<0.01 ~}$ vs. sham group; ${ }^{*} \mathrm{P}<0.05,{ }^{* *} \mathrm{P}<0.01$ vs. angII model group. Pue, puerarin; MMP-2, matrix metalloproteinase 2; AP-1, activator protein-1; p-Jun, phosphorylated Jun; angII, angiotensin II.

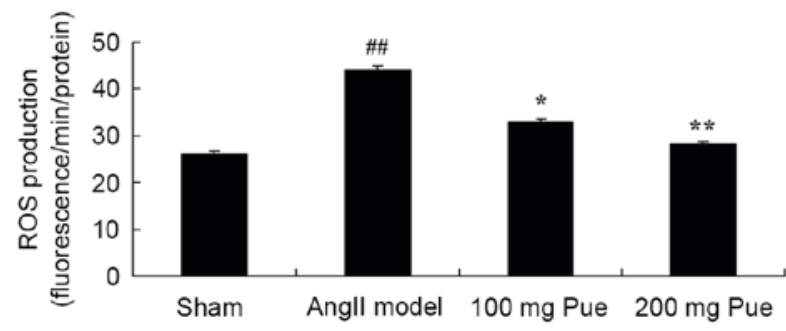

Figure 5. Pue treatment suppressed ROS production in angII-induced abdominal aortic aneurysm in mice. ${ }^{\# \#} \mathrm{P}<0.01$ vs. sham group; ${ }^{*} \mathrm{P}<0.05,{ }^{* *} \mathrm{P}<0.01$ vs. angII model group. Pue, puerarin; ROS, reactive oxygen species; angII, angiotensin II.

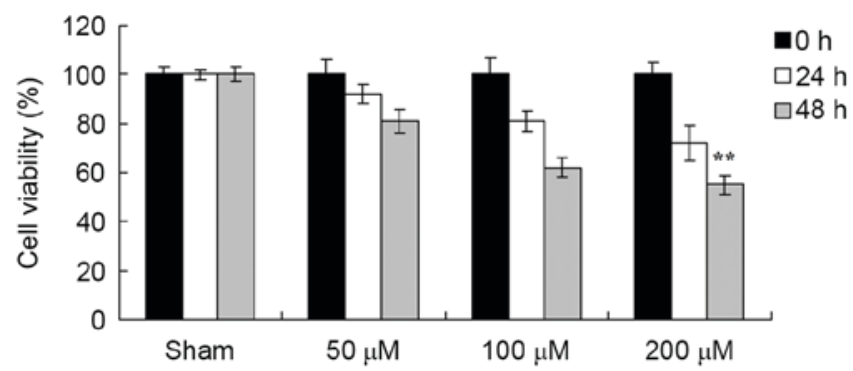

Figure 6. Pue treatment attenuated the viability of murine hemangioendothelioma cells. Cell viability was determined using an MTT assay following treatment with dimethyl sulfoxide (sham) or the indicated concentrations of Pue, for 0,24 or 48 h. ${ }^{*} \mathrm{P}<0.05,{ }^{* *} \mathrm{P}<0.01$ vs. sham group. Pue, puerarin.

particular, 100 and $200 \mu \mathrm{M}$ puerarin significantly induced the apoptosis of EOMA cells at $48 \mathrm{~h}$ (Fig. 7).

Puerarin induces increased caspase-9 and -3 activity in the EOMA cells. To determine the effect of puerarin on the caspase- 9 and -3 activity of EOMA cells, caspase- 9 and caspase- 3 were measured using a chromogenic assay. As presented in Fig. 8, 100 and $200 \mu \mathrm{M}$ puerarin significantly

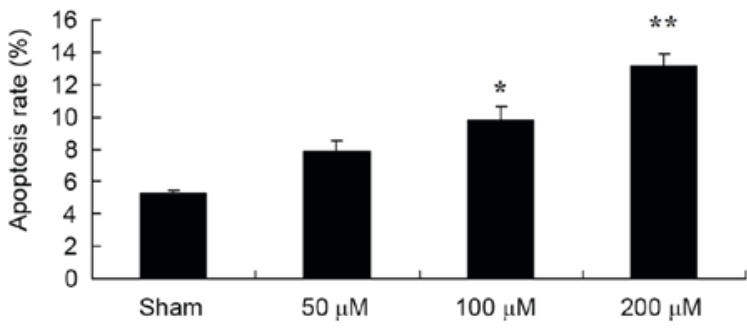

Figure 7. Pue treatment induced the apoptosis of murine hemangioendothelioma cells. Flow cytometric analysis was performed following treatment with dimethyl sulfoxide (sham), or the indicated concentrations of Pue. ${ }^{*} \mathrm{P}<0.05,{ }^{* *} \mathrm{P}<0.01$ vs. control. Pue, puerarin.

increased the activities of caspase- 9 and caspase-3 in EOMA cells.

\section{Discussion}

During the development of AAA, a large number of ROS will be generated and inflammatory cells will release cytokines, damaging the normal structure of the vascular wall (4). The expression level of ROS and reactive nitrogen species (RNS) in human AAA specimens is significantly increased (16). A previous study demonstrated that the oxidative stress reaction in the aorta wall is associated with the incidence of AAA; it can prevent the formation of atherosclerosis (16). Oxidative stress may promote the formation of AAA through the regulation of p-Jun/AP-1 (8). To the best of our knowledge, the present study was the first to demonstrate that puerarin significantly decreased the angII-induced AAA rate in mice and suppressed viability, induced apoptosis and increased the caspases 9 and 3 activity in EOMA cells. Zhang et al (17) revealed that puerarin inhibits growth and induces apoptosis of hepatocellular carcinoma cells. In addition, Yu et al (13) indicated that puerarin induced apoptosis and decreased the viability of colon cancer cells. 

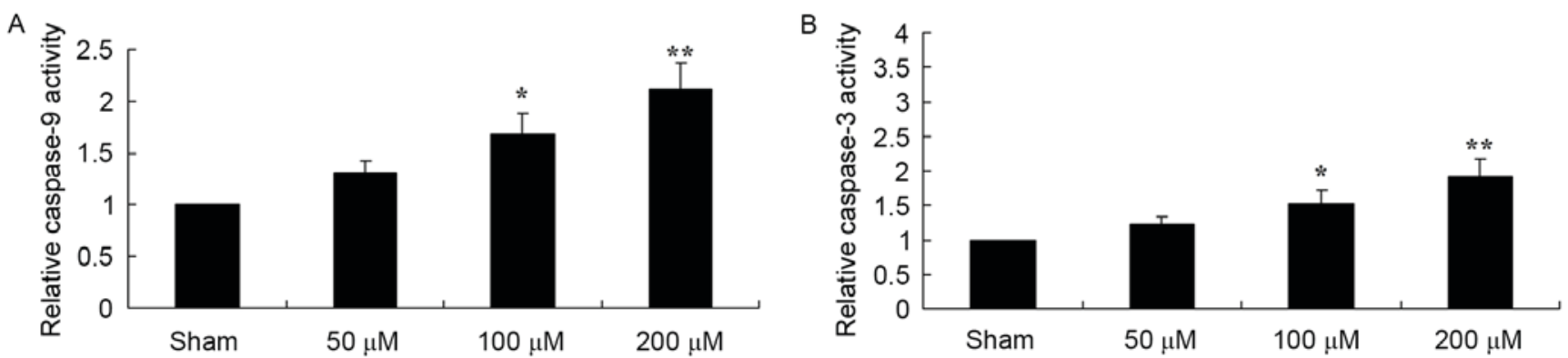

Figure 8. Pue treatment induced increased caspase-9 and -3 activity in murine hemangioendothelioma cells. The activity of (A) caspase- 9 and (B) caspase-3 was determined using a chromogenic assay following treatment with dimethyl sulfoxide (sham), or the indicated concentrations of Pue. ${ }^{*} \mathrm{P}<0.05,{ }^{* *} \mathrm{P}<0.01 \mathrm{vs}$. control. Pue, puerarin.

NADPH oxidase's substrates are $\mathrm{O}_{2}$ and NADPH. The catalytic reaction process may generate a large amount of $\mathrm{O}_{2}^{-}$; NADPH oxidase is the principal source of ROS in the vascular wall (18). The specific inhibitor of NADPH oxidase, acetovanillone, was determined to inhibit AAA in a mouse model (19). NADPH oxidase requires membrane coupling and accessory proteins (including Rac, p47phox and p67phox) to catalyze the generation of superoxide (20). It was previously identified that quercetin downregulated the expression of $\mathrm{p} 47$ phox in vitro and in vivo, decreasing the generation of $\mathrm{O}_{2}$ in the aorta (20). Following the gene knockout of p47phox, oxidative stress levels are alleviated and the formation of AAA is inhibited (19). Furthermore, the results of the present study demonstrated that puerarin significantly decreased the angII-induced NADPH oxidase activity in AAA in mice. Kim et al (14) demonstrated that puerarin inhibits the apoptosis of retinal pericytes by inhibiting NADPH oxidase-related oxidative stress.

Oxidative stress is associated with the pathological process of the inflammatory reaction. Oxidative stress is tissue damage incurred subsequent to ROS increase and/or depletion (21). There is a balance between the generation of ROS (including NADPH oxidase) with antioxidants and antioxidant enzymes (22). It is hypothesized that ROS and oxidative stress may be associated with human AAA pathogenesis; ROS and RNS are increased in AAA tissue (22). The results of the present study demonstrated that puerarin treatment significantly decreased the production of ROS in angII-induced AAA mice. Zhang et al (23) previously identified that puerarin attenuates cognitive dysfunction and oxidative stress by ROS generation in a rat model of vascular dementia.

The balance between MMPs and their inhibitors is required to maintain the integrity of the arterial wall structure (24). MMP-2 and -9 are predominantly expressed in AAA tissue. A previous study has identified that MMP regulation may serve a protective role in AAA; for example, mice lacking MMP-2 or -9 did not form AAA (25). Oxidative stress is an important regulator of MMPs; ROS may activate MMPs and induce the degradation of the extracellular matrix. In thoracic aortic aneurysms, the MMP activity is enhanced subsequent to NADPH oxidase overexpression (26). In addition, in a mouse model with thin arteries and veins, an increase in silicate increased the activity of MMP-2 and -9 , and induced vascular remodeling (27). In the present study, puerarin treatment significantly inhibited the expression of MMP-2 protein in
angII-induced AAA mice. Yang et al (28) demonstrated that puerarin decreased alveolar bone loss and collagen destruction by inhibiting the production of MMP-2 and MMP-9 in rats.

The stress response in various cell types activates c-Jun $\mathrm{N}$-terminal kinases (JNKs). For this reason, JNKs are also known as stress-activated protein kinases (29). It has been established that ROS and RNS stimulate the production of NADPH. It has also been demonstrated that JNKs are important in the incidence of AAA. Through drug inhibition, it has been demonstrated that JNKs may decrease the activity of MMPs and prevent the formation of AAA (30). JNKs may regulate the phosphorylation and nuclear translocation of transcription factors, including AP-1, and other kinases (31). Gang et al (10) demonstrated that puerarin suppressed angII-induced cardiac hypertrophy by the AP-1 and JNK1/2 signaling pathways. In the present study, puerarin significantly inhibited AP-1 protein expression and increased the p-Jun protein expression in AAA in mice. Thus, puerarin treatment may reduce the oxidative stress level and prevent the development of AAA via the regulation of the JNK/AP-1 pathway and MMPs.

In conclusion, in the present study, it was demonstrated that puerarin significantly decreased the angII-induced AAA rate in a mouse model, suppressed viability, induced apoptosis, and increased the activity of caspase- 9 and -3 in EOMA cells. This may have been due to the inhibition of NADPH oxidase activation and oxidative stress-triggered AP-1 signaling pathways.

\section{Acknowledgements}

Not applicable.

\section{Funding}

No funding received.

\section{Availability of data and materials}

The analyzed data sets generated during the study are available from the corresponding author on reasonable request.

\section{Authors' contributions}

JH designed the experiment, JY, SC, ZX and YQ performed the experiments, JH and JY analyzed the data and JH wrote the manuscript. 


\section{Ethics approval and consent to participate}

All protocols in the present study were approved by the Ethics Committee of the Hainan Provincial People's Hospital (Guangzhou, China).

\section{Patient consent for publication}

Not applicable.

\section{Competing interests}

The authors declare that they have no competing interests.

\section{References}

1. Lyck Hansen M, Dahl Thomsen M, Rasmussen LM and Lindholt JS: Abdominal aortic aneurysm, arterial stiffening and the role of the intraluminal thrombus. Vasa 44: 349-353, 2015.

2. Barakat HM, Shahin Y, Barnes R, Gohil R, Souroullas P, Khan J, McCollum PT and Chetter IC: Supervised exercise program improves aerobic fitness in patients awaiting abdominal aortic aneurysm repair. Ann Vasc Surg 28: 74-79, 2014.

3. Nienaber CA, Kische S, Rousseau H, Eggebrecht H, Rehders TC Kundt G, Glass A, Scheinert D, Czerny M, Kleinfeldt T, et al Endovascular repair of type B aortic dissection: long-term results of the randomized investigation of stent grafts in aortic dissection trial. Circ Cardiovasc Interv 6: 407-416, 2013.

4. United Kingdom EVAR Trial Investigators, Greenhalgh RM, Brown LC, Powell JT, Thompson SG and Epstein D: Endovascular repair of aortic aneurysm in patients physically ineligible for open repair. N Engl J Med 362: 1872-1880, 2010

5. Wong YY, Flicker L, Yeap BB, McCaul KA, Hankey GJ and Norman PE: Is hypovitaminosis D associated with abdominal aortic aneurysm, and is there a dose-response relationship? Eur J Vasc Endovasc Surg 45: 657-664, 2013.

6. Cafueri G, Parodi F, Pistorio A, Bertolotto M, Ventura F, Gambini C, Bianco P, Dallegri F, Pistoia V, Pezzolo A and Palombo D: Endothelial and smooth muscle cells from abdominal aortic aneurysm have increased oxidative stress and telomere attrition. PLoS One 7: e35312, 2012.

7. Guzik B, Sagan A, Ludew D, Mrowiecki W, Chwała M, Bujak-Gizycka B, Filip G, Grudzien G, Kapelak B, Zmudka K, et al: Mechanisms of oxidative stress in human aortic aneurysms-association with clinical risk factors for atherosclerosis and disease severity. Int J Cardiol 168: 2389-2396, 2013.

8. Kaneko H, Anzai T, Horiuchi K, Kohno T, Nagai T, Anzai A, Takahashi T, Sasaki A, Shimoda M, Maekawa Y, et al: Tumor necrosis factor- $\alpha$ converting enzyme is a key mediator of abdominal aortic aneurysm development. Atherosclerosis 218: 470-478, 2011.

9. Sawada H, Hao H, Naito Y, Oboshi M, Hirotani S, Mitsuno M, Miyamoto Y, Hirota S and Masuyama T: Aortic iron overload with oxidative stress and inflammation in human and murine abdominal aortic aneurysm. Arterioscler Thromb Vasc Biol 35: 1507-1514, 2015.

10. Gang C, Qiang C, Xiangli C, Shifen P, Chong S and Lihong L: Puerarin suppresses angiotensin ii-induced cardiac hypertrophy by inhibiting nadph oxidase activation and oxidative stress-triggered ap-1 signaling pathways. J Pharm Pharm Sci 18: 235-248, 2015.

11. Liu X, Mo Y, Gong J, Li Z, Peng H, Chen J, Wang Q, Ke Z and Xie J: Puerarin ameliorates cognitive deficits in streptozotocin-induced diabetic rats. Metab Brain Dis 31: 417-423, 2016.

12. Gao Z, Wei B and Qian C: Puerarin injection for treatment of unstable angina pectoris: A meta-analysis and systematic review. Int J Clin Exp Med 8: 14577-14594, 2015.

13. $\mathrm{Yu} \mathrm{Z}$ and $\mathrm{Li} \mathrm{W}$ : Induction of apoptosis by puerarin in colon cancer HT-29 cells. Cancer Lett 238: 53-60, 2006.

14. Kim J, Kim KM, Kim CS, Sohn E, Lee YM, Jo K and Kim JS: Puerarin inhibits the retinal pericyte apoptosis induced by advanced glycation end products in vitro and in vivo by inhibiting NADPH oxidase-related oxidative stress. Free Radic Biol Med 53: 357-365, 2012.
15. Ren H, Li F, Tian C, Nie H, Wang L, Li HH and Zheng Y: Inhibition of proteasome activity by low-dose bortezomib attenuates angiotensin ii-induced abdominal aortic aneurysm in apoe(-/-) mice. Sci Rep 5: 15730, 2015.

16. Blogowski W, Dolegowska B, Pikula E, Gutowski P and Starzynska T: The effect of PGE administration on the activity of oxidative system in erythrocytes and platelets during ischemia reperfusion injury and on postoperative renal function in patients undergoing open abdominal aortic aneurysm reconstruction. J Biol Regul Homeost Agents 26: 429-438, 2012.

17. Zhang WG, Liu XF, Meng KW and Hu SY: Puerarin inhibits growth and induces apoptosis in SMMC-7721 hepatocellular carcinoma cells. Mol Med Rep 10: 2752-2758, 2014.

18. Liu Z, Luo H, Zhang L, Huang Y, Liu B, Ma K, Feng J, Xie J, Zheng J, Hu J, et al: Hyperhomocysteinemia exaggerates adventitial inflammation and angiotensin II-induced abdominal aortic aneurysm in mice. Circ Res 111: 1261-1273, 2012.

19. Thomas M, Gavrila D, McCormick ML, Miller FJ Jr, Daugherty A, Cassis LA, Dellsperger KC and Weintraub NL: Deletion of p47phox attenuates angiotensin II-induced abdominal aortic aneurysm formation in apolipoprotein E-deficient mice. Circulation 114: 404-413, 2006.

20. Miao XN, Siu KL and Cai H: Nifedipine attenuation of abdominal aortic aneurysm in hypertensive and non-hypertensive mice: Mechanisms and implications. J Mol Cell Cardiol 87: 152-159, 2015.

21. Zhang H, Wang ZW, Wu HB, Li Z, Li LC, Hu XP, Ren ZL, Li BJ and Hu ZP: Transforming growth factor- $\beta 1$ induces matrix metalloproteinase- 9 expression in rat vascular smooth muscle cells via ROS-dependent ERK-NF-кB pathways. Mol Cell Biochem 375: 11-21, 2013.

22. Yan H, Cui B, Zhang X, Fu X, Yan J, Wang X, Lv X, Chen Z and $\mathrm{Hu} Z$ : Antagonism of toll-like receptor 2 attenuates the formation and progression of abdominal aortic aneurysm. Acta Pharm Sin B 5: 176-187, 2015.

23. Zhang J, Guo W, Tian B, Sun M, Li H, Zhou L and Liu X: Puerarin attenuates cognitive dysfunction and oxidative stress in vascular dementia rats induced by chronic ischemia. Int J Clin Exp Pathol 8: 4695-4704, 2015

24. Dale MA, Suh MK, Zhao S, Meisinger T, Gu L, Swier VJ, Agrawal DK, Greiner TC, Carson JS, Baxter BT and Xiong W: Background differences in baseline and stimulated MMP levels influence abdominal aortic aneurysm susceptibility. Atherosclerosis 243: 621-629, 2015.

25. Rabkin SW: Differential expression of MMP-2, MMP-9 and TIMP proteins in thoracic aortic aneurysm - comparison with and without bicuspid aortic valve: a meta-analysis. Vasa 43: 433-442, 2014.

26. Zhang T, Xu J, Li D, Chen J, Shen X, Xu F, Teng F, Deng Y, Ma H, Zhang L, et al: Salvianolic acid A, a matrix metalloproteinase-9 inhibitor of Salvia miltiorrhiza, attenuates aortic aneurysm formation in apolipoprotein E-deficient mice. Phytomedicine 21: 1137-1145, 2014.

27. Deguchi JO, Huang H, Libby P, Aikawa E, Whittaker P, Sylvan J, Lee RT and Aikawa M: Genetically engineered resistance for MMP collagenases promotes abdominal aortic aneurysm formation in mice infused with angiotensin II. Lab Invest 89: 315-326, 2009.

28. Yang X, Zhang H, Wang J, Zhang Z and Li C: Puerarin decreases bone loss and collagen destruction in rats with ligature-induced periodontitis. J Periodontal Res 50: 748-757, 2015.

29. Wang C, Chang Q, Qian X, Tian C and Sun X: Angiotensin II induces an increase in MMP-2 expression in idiopathic ascending aortic aneurysm via AT1 receptor and JNK pathway. Acta Biochim Biophys Sin (Shanghai) 47: 539-547, 2015.

30. DiMusto PD, Lu G, Ghosh A, Roelofs KJ, Sadiq O, McEvoy B, Su G, Laser A, Bhamidipati CM, Ailawadi G, et al: Increased JNK in males compared with females in a rodent model of abdominal aortic aneurysm. J Surg Res 176: 687-695, 2012.

31. Wang L, Cheng X, Li H, Qiu F, Yang N, Wang B, Lu H, Wu H, Shen Y, Wang Y and Jing H: Quercetin reduces oxidative stress and inhibits activation of cJun Nterminal kinase/activator protein1 signaling in an experimental mouse model of abdominal aortic aneurysm. Mol Med Rep 9: 435-442, 2014. 